\title{
Über analytische Anwendungen der Uramidosäurereaktion.
}

\author{
Von
}

Priv.-Doz. Dr. F. Lippich.

(Aus dem mediz.-chem. Institute der Prager deutschen Universitāt.)

(Der Redaktion zugegangen am 20. Februar 1914.)

In einer ersten kurzen Mitteilung ${ }^{1}$ ) habe ich darauf hingewiesen, daß die von mir später verallgemeinerte Bildungsweise der Uramidosäuren, Kochen von Amidosäure mit Harnstoff und Barytwasser, mit Vorteil zum Nachweis kleiner Mengen von Leucin verwendet werden kann. Damals habe ich angegeben, daß durch Überführung in die Uramidosäure auf dem genannten Wege noch $0,02 \mathrm{~g}$ Leucin mit Sicherheit nachzuweisen sind. Ich konnte dann weiterhin ${ }^{2}$ ) zeigen, daß noch andere einfache Bildungsweisen der Uramidosäuren existieren, von denen besonders jene; bei welcher schon durch bloßes Kochen von Amidosäure mit Wasser und Harnstoff allein Uramidosäure gebildet wird, analytisches Interesse beansprucht.

Zunächst gelingt es mit Hilfe dieser Bildungsweise, den Leucinnachweis, $d . h$. den Nachweis, daß ein vorliegender isolierter Körper Leucin ist, oder Leucin enthält, sehr viel empfindlicher zu gestalten, als es mit der erstangeführten Bildungsweise möglich ist. Es wurde schon seinerzeit ausgeführt, daß die Entstehung von Uramidosäure beim Kochen mit reinem Wasser darauf beruht, $\mathrm{da}$ in einer wässerigen Harnstofflösung stets ein Gleichgewicht zwischen Harnstoff und Ammoniumcyanat existiert; das Ammoniumcyanat wird von der gegenwärtigen Aminosäure weggefangen, und diese

1) B. B., Bd. 39, S. 2953, 1906.

2) Ebenda, Bd. 41, S. 2974, 1908. 
Störung des Gleichgewichtes führt solange zu einer Umwandlung von Harnstoff in Ammoniumcyanat resp. zu einer Bildung von Uramidosäure, bis ein neuer Gleichgewichtszustand sich eingestellt hat, der umsomehr nach der Seite der Uramidosäure verschoben sein wird, je günstiger die Temperatur- und Konzentrationsverhältnisse liegen. Zum Zwecke der analytischen Verwertung muß also das Gleichgewicht möglichst nach der Uramidosäureseite verschoben werden, da ja hiervon die Empfindlichkeit abhängt.

Da bei sehr kleinen Leucinmengen ein Erfolg nur dann zu erwarten war, wenn die Konzentration an Aminosäure möglichst groß, die Wassermenge also möglichst klein gemacht wurde, so wurde wie folgt vorgegangen: In einer kleinen Eprouvette von 10-12 ccm Inhalt und 10-12 mm Durchmesser werden wenige Milligramm der zu untersuchenden Substanz abgewogen; sodann wird die mehrfache Gewichtsmenge Harnstoff und je nach der Substanzmenge $2-1-1 / 2 \mathrm{ccm}$ Wasser hinzugefügt; nun versieht man die Eprouvette mit einem nicht $\mathrm{zu}$ engen am unteren Ende abgeschrägten Steigrohr und erhitzt mit einem kleinen Flämmchen zum Sieden; eventuelles Stoßen wird in bekannter Weise vermieden; man erhält je nach der Substanzmenge $1 / 4-1 / 2-1-11 / 2$ Stunden im Sieden; sodann wird nach dem Abkühlen vorsichtig mit verdünnter Mineralsäure angesäuert; unter häufigem Schütteln erfolgt, wenn Leucin vorlag, die typische Krystallisation, mitunter erst nach mehrstündigem Stehen; es ist zweckmäßig, nach dem Ansäuern zu impfen; zu großer Harnstoffüberschuß hemmt die Krystallisation.

Einige Versuche mögen das Angeführte erläutern:

I. Je $4 \mathrm{mg}$ Leucin wurden in der angegebenen Weise mit $1 \mathrm{ccm}$ Wasser das eine Mal $1 / 4$, das andere Mal ${ }^{1 / 2}$ Stunde gekocht; nach dem Abkühlen und Ansäuern trat in beiden Fällen alsbald die charakteristische, aus Nadeln bestehende Krystallisation der Leucinursäure auf, im zweiten Falle wesentlich stärker als im ersten.

II. Je $3 \mathrm{mg}$ Leucin; $1 \mathrm{ccm}$ Wasser; Kochdauer $1 / 2$ und 1 Stunde; nach Impfen und längerem Schütteln mikro- 
krystallinischer Niederschlag; nach 12 Stunden lange Krystallnadeln. Im zweiten Falle krystallinischer Niederschlag alsbald nach dem Ansäuern.

III. Je $2 \mathrm{mg}$ Leucin; $1 \mathrm{ccm}$ Wasser; Kochdauer $1 / 2$ und 1 Stunde; im ersten Falle nach Impfen und häufigem Schütteln keine deutliche Reaktion; im zweiten Falle tritt nach einiger Zeit die charakteristische Krystallisation auf.

IV. Je $1 \mathrm{mg}$ Leucin; 1 und $1 / 2$ ccm Wasser; Kochdauer 1 und $11 / 2$ Stunden. Im ersten Falle keine positive Reaktion; im zweiten Falle tritt nach einiger Zeit eine schwache, aber deutliche Reaktion auf.

Wenn also in der angegebenen Weise sich die Empfindlichkeit der Reaktion wenigstens für reines Leucin recht bedeutend steigern läßt, so muß doch darauf aufmerksam gemacht werden, daß dieselbe noch von anderen Faktoren abhängt; insbesondere von jener verschiedenen Löslichkeit, welche die Uramidosäuren aus Leucinen verschiedener Herkunft aufweisen, worauf schon seinerzeit nachdrücklich hingewiesen wurde. ${ }^{1}$ )

Daß diese Reaktion infolge ihrer Empfindlichkeit, also auch infolge der kleinen Substanzmengen, mit welchen noch sichere Resultate erhalten werden können, infolge der Einfachheit und Schnelligkeit der Ausführung, vielfacher Anwendungen fähig ist, braucht wohl kaum besonders hervorgehoben resp. durch Beispiele erhärtet zu werden; dennoch seien hier solche angeführt.

Gelegentlich einer einfachen Darstellung von Leucin aus Hämoglobin durch Säurespaltung war nach dem Umkrystallisieren der Leucin-Tyrosinfraktion aus ammoniakalischem Alkohol ein dunkelbrauner Rückstand erhalten worden, der eine starke Tyrosinreaktion gab. Die in obiger Weise mit einer kleinen Menge des Rückstandes angestellte Reaktion ergab sofort dessen reichlichen Leucingehalt. Infolgedessen wurden $10 \mathrm{~g}$ obiger Rückstände mit $20 \mathrm{~g}$ Harnstoff und kalt gesättigtem Barytwasser 15 Stunden gekocht, der überschüssige Baryt mit Kohlensäure entfernt und das eingeengte, schwach

1) B. B., Bd. 41, S. 2953, 1908. 
gelbe Filtrat mit Essigsäure gefällt. Diese erste Fällung betrug 6,7 g und war fast farblos. Sie wnrde einmal aus 50\% igem Alkohol umkrystallisiert. Die nunmehr rein weißen Krystalle gaben bei der Stickstoffbestimmung die folgenden Werte:

$0,2242 \mathrm{~g}$ Substanz banden $25,57 \mathrm{ccm} \mathrm{n} / 10-\mathrm{H}_{2} \mathrm{SO}_{4}$. $0,3043 \mathrm{~g}$ Substanz $44,0 \mathrm{ccm} \mathrm{N}\left(20,4^{0} ; 723,4 \mathrm{~mm}\right)$.

$$
\begin{array}{ll}
\mathrm{C}_{7} \mathrm{H}_{14} \mathrm{~N}_{2} \mathrm{O}_{3} \text {. } & \text { Ber.: } \mathrm{N}=16,091 \% . \\
& \text { Gef.: } \mathrm{N}=15,968 \%, 16,286 \% .
\end{array}
$$

Aus diesem Beispiele kann zugleich entnommen werden, mit welch großem Vorteile die Uramidosäure zur raschen Isolierung und Reindarstellung von Leucin in Form der Hydantoinsäure verwendet werden kann, wobei nicht zuletzt die Einfachheit der Ausführung gegenüber den anderen Methoden ins Gewicht fällt. Darüber wird noch an andrer Stelle berichtet werden.

Als weiteres Beispiel sei das folgende angeführt:

Es handelte sich darum, rasch zu entscheiden, ob ein vorliegendes Präparat, das dem Stickstoffwerte und der spezifischen Drehung nach als unreines Valin hätte gelten können, größere Mengen Leucin enthalte. Die mit einer kleinen Menge des fraglichen Präparates ausgeführte Leucinreaktion zeigte unzweifelhaft die Anwesenheit einer größeren Menge dieses Körpers. Durch Kochen des so geprüften Präparates mit Harnstoff und Barytwasser konnte das Leucin leicht zum größten Teile von der beigemengten Substanz in Form der Uramidosäure getrennt werden. Nach einmaligem Umkrystallisieren aus 50\%igem Alkohol zeigte die letztere einen Schmelzpunkt von $188^{\circ}$ (im geschlossenen Kapillarrohr) und einen Stickstoffgehalt von $15,8 \%$ (nach Kjeldah]).

Wo es angeht, wird man die geschilderte Reaktion mit so großen Mengen ausführen, daß man eventuell eine Isolierung des erhaltenen krystallinischen Niederschlages zum Zwecke einer Schmelzpunktbestimmung durchführen kann. Jch sehe mich bei dieser Gelegenheit neuerdings veranlaßt, nachdrücklichst darauf hinzuweisen, daß der von mir in meiner ersten Mitteilung ${ }^{1}$ ) angegebene Schmelzpunkt der Leucinursäure für

1) B. B., Bd. 39, S. 2953, 1906. 
offenes Kapillarrohr gilt, während in den folgenden Mitteilungen ${ }^{1}$ ) die Schmelzpunkte derUramidosäuren durchaus für geschlossenes Kapillarrohr angegeben sind. Ich habe damals und auch später ${ }^{2}$ ) die Gründe auseinandergesetzt, warum ich die Bestimmung im geschlossenen Kapillarrohre ausführe. Trotzdem findet es Weil and ${ }^{3}$ ) auffällig, daß ich in meiner ersten Mitteilung einen höheren Schmelzpunkt für die Leucinursäure angegeben habe, als in meinen späteren Mitteilungen. $\mathrm{Da}$ er für racemische Leucinursäure einen ähnlichen Schmelzpunkt im off enen Kapillarrohr findet wie ich für meine Leucinursäurepräparate im geschlossenen, so folgert er daraus, daß die Differenzen in den Schmelzpunkten, die ich bei den Leucinursäuren aus Leucinen verschiedener Herkunft gefunden habe, auf verschiedene Racemisierungsgrade zurückzuführen sind und nicht, wie ich seinerzeit ausführte, möglicherweise auf eine verschiedenartige Zusammensetzung der Ausgangsmaterialien aus Leucinen verschiedener Konstitution. Die Bemerkungen Weilands über diesen Punkt erledigen sich wohl durch den Hinweis, daß Schmelzpunktbestimmungen für offenes und geschlossenes Kapillarrohr nicht ohne weiteres vergleichbar sind und daß naturgemäß für geschlossenes Kapillarrohr der Schmelzpunkt der racemischen Leucinursäure hätte wesentlich tiefer ausfallen müssen.

Auch wenn eine Schmelzpunktbestimmung nicht angeschlossen werden kann, so ist doch die Reaktion für Leucin so charakteristisch und beweisend, als es etwa die Biuretreaktion für Harnstoff, oder die Murexidprobe für Harnsäure ist, zum mindesten soweit es sich um physiologische Anwendungen handelt. Denn einmal wird ja doch der entscheidenden Reaktion, wenn das Leucin bis zu einem gewissen Grade isoliert vorliegt, eine gewisse Abgrenzung und Vorprüfung vorangehen, zweitens ist mir keine physiologisch vorkommende Substanz bekannt, von anderen Aminosäuren zunächst abgesehen; die mit Harnstoff in wässeriger Lösung unter Bildung eines schwerlöslichen Produktes reagieren würde, und sollte

1) B. B., Bd. 41, S. 2953 und 2974, 1908.

2) Diese Zeitschrift, Bd. 68, S. 278, 1910.

3) Biochem. Zeitschr., Bd. 38, S. 385, 1912. 
endlich.schon ja einmal eine Verwechslung mit schwerlöslichen organischen Säuren, wie Benzoesäure, Salicylsäure, Hippursäure usw., möglich sein, so läßt sich bei den kleinen Mengen, die in Betracht kommen, leicht eine Entscheidung mit Hilfe eines organischen Lösungsmittels wie Äther, Petroläther, Essigäther usw. herbeiführen, in welchen die Leucinursäure nicht oder sehr wenig löslich ist. Ernstlich in Frage käme nur dort, wo sich eine Schmelzpunktbestimmung nicht ausführen läßt, die Möglichkeit einer Verwechslung mit anderen Aminosäuren resp. Uramidosäuren. Zunächst wäre an erster Stelle das Valin resp. die Valinursäure zu berücksichtigen. Die letztere besitzt nach meinen Bestimmungen eine Löslichkeit in Wasser von ca. 1 : 200; $1 \mathrm{ccm}$ Wasser löst daher $5 \mathrm{mg}$; angenommen nun, ein vorliegender Körper sei reines Valin und es werden wie unter Versuch I., S. 125, $4 \mathrm{mg}$ in $1 \mathrm{ccm}$ Wasser mit Harnstoff gekocht, so würden, vorausgesetzt, daß eine quantitative Umwandlung erfolgt, 5,5 mg Valinursäure gebildet werden. Selbst also in diesem angenommenen Falle würde eine Krystallisation von Valinursäure kaum erfolgen können, umsoweniger in Wirklichkeit, da ja die Reaktion nicht quantitativ ist. Man wird also durch entsprechende Versuchsanordnung eine Verwechslung mit dieser Uramidosäure stets vermeiden können und es erweist sich im Gegenteil, wie hier schon hervorgehoben, an anderer Stelle aber noch in anderer Beziehung erörtert werden soll, die Reaktion sehr nützlich, um neben viel Valin relativ kleine Mengen von Leucin zu entdecken.

Etwas weniger günstig liegt die Sache bei der Uramidosäure des Phenylalanins, der Phenylalanursäure, insofern, als diese Säure nach der Leucinursäure die schwerlöslichste der bisher bekannten Uramidosäuren ist; ihre Wasserlöslichkeit beträgt ca. 1 : 600; da die Löslichkeit der Leucinursäure mindestens $1: 1600$ beträgt, so ließe sich auch hier durch entsprechende Versuchsanordnung eine Verwechslung vermeiden, doch wird es für alle Fälle sicherer sein, der Leucinursäurereaktion die bekannte Reaktion auf Phenylalanin, die genügende Empfindlichkeit besitzt, voranzuschickèn, oder aber dieselbe mit dem Uramidosäureniederschlag nachträglich anzustellen.

Hoppe-Seyler's Zeitschrift f. physiol. Chemie. XC. 
Da sich späterhin kaum mehr die passende Gelegenheit dazu bieten dürfte, so sei hier einiges über die Uramidosäure des Phenylalanins und deren Anhydrid eingeschaltet.

Die Uramidosäure, die $\alpha$-Ureido- $\beta$-phenylpropionsäure oder die Phenylalanursäure wurde von $\mathrm{Dakin}^{1}$ ) gewonnen, nachdem er einer Katze $8 \mathrm{~g}$ Phenylalanin in die Femoralvene injiziert hatte. Beim Stehen des Harns schied sich direkt aus demselben eine geringe Menge von Krystallen $a b$; eine weitere Menge Substanz wurde aus dem angesäuerten Harn durch Extraktion mit Äthylacetat gewonnen. Nur letztere wurde offenbar analysiert und zeigte einen auf die Uramidosäure stimmenden Stickstoffgehalt und einen mit jenem der aus Phenylalanin und Kaliumcyanat dargestellten Säure übereinstimmenden Schmelzpunkt von $188-190^{\circ}$ (im offenen Kapillarrohr). Das direkte Ausfallen eines Teiles der Uramidosäure aus dem Harn ist um so auffallender, als doch bekanntlich der Säugerorganismus eine ziemliche Menge von Ammoniak zur Neutralisation von Säuren aufzubringen vermag, und in diesem Falle außerdem noch die einem großen Teile des eingeführten Phenylalanins entsprechende Ammoniakmenge zur Verfügung stand. Man möchte eher schließen, daß hier nicht freie Säure, sondern Anhydrid vorlag, welches sich bei der Phenylalanursäure ebenso leicht bildet wie bei der Tyrosinursäure, und welches im Wasser sehr schwer löslich ist. Es sei dies deshalb hervorgehoben, weil Dakin mit Rücksicht auf die früheren Beobachtungen über Uramidosäurebildung im Organismus es besonders bemerkenswert findet, daß die Substanz aus einem Harn ausfiel, der keinen Prozeduren irgendwelcher Art unterworfen worden war. Im Hinblick auf die von mir gemachten Beobachtungen über die Bildung von Uramidosäuren in wässeriger Lösung wird selbstverständlich beim bloßen Eindampfen einer Harnstoff- und Aminosäure enthaltenden Lösung Uramidosäure entstehen müssen, je nach der Temperatur in größerem oder geringerem Umfange und niemals bei Gegenwart freier Säure, selbst Essigsäure nicht, was übrigens den Bildungsweisen der Uramidosäuren gemäß selbstverständlich erscheint, so daß diesbezüglich kaum be-

1) Journ. of Biol. chem., Bd. 6, S. 235, 1909. 
sondere.Versuche notwendig sind, wie sie Dakin mitteilt. In besonders breiter Form 'glaubt Weiland diese Verhältnisse hervorheben zu müssen. Die von Dakin aus diesen Beobachtungen abgeleitete Folgerung, daß nur die von ihm beschriebene Uramidosäurebildung unter den bisher beobachteten die einzige sei, welche die Fähigkeit des Organismus zu dieser Synthese sicher beweise, scheint mir etwas zu weit gegangen $\mathrm{zu}$ sein.

Wie schon erwähnt, benutzt Dakin zur synthetischen Darstellung der Phenylalanursäure die gewöhnlich verwendete Methode der Kombination von Aminosäure mit Kaliumcyanat. Von mir wurde die Säure nach der seinerzeit mitgeteilten Methode durch Kochen von Phenylalanin mit Harnstoff und Barytwasser dargestellt. $1 \mathrm{~g}$ der Aminosäure wurde mit $3 \mathrm{~g}$ Harnstoff in gesättigtem Barytwaser durch 4 Stunden erhitzt. Nach Entfernung des überschüssigen Baryts mit Kohlensäure und Eindampfen des Filtrates wurde ein allmählich in Nadeln krystallisierendes, in Wasser sehr leicht lösliches Barytsalz erhalten. Durch Ansäuren seiner wässerigen Lösung mit verdünnter Salzsäure fiel die Phenylalanursäure in Büscheln und Rosetten ziemlich breiter und derber Nadeln aus, die eine gewisse Ähnlichkeit mit Albuminkrystallen zeigten. Es wurden ca. 0,4 g Substanz erhalten; ihre Löslichkeit in Wasser von ca. $20^{\circ}$ betrug, wie schon angeführt, etwa $1: 600$; die Löslichkeit in wasserhaltigem Äther 1:4000; der Schmelzpunkt im geschlossenen Kapillarrohr lag bei $175^{\circ} ; 0,1529$ g Substanz $17,6 \mathrm{ccm} \mathrm{N}\left(15,6^{\circ}: 746 \mathrm{~mm}\right)$.

$$
\begin{aligned}
& \mathrm{C}_{10} \mathrm{H}_{12} \mathrm{~N}_{2} \mathrm{O}_{3} . \quad \text { Ber.: } \mathrm{N}=13,46 \% . \\
& \text { Gef.: } \mathrm{N}=13,42 \% .
\end{aligned}
$$

Das Filtrat von der Fällung mit Salzsäure wurde mit dieser gekocht; nach dem Erkalten bildete sich alsbald ein krystallinischer Niederschlag, neben dem nach einiger Zeit schöne, relativ große, regelmäßige Krystalle auftraten. Die so erhaltene Substanz ist das bisher noch nicht dargestellte Anhydrid der Phenylalanur- oder $\alpha$-Uramido- $\beta$-phenylpropionsäure. Der Körper ist in Wasser sehr schwer, in Äther merklich löslich, so daß er mit Äther ausgeschüttelt werden kann. Er-

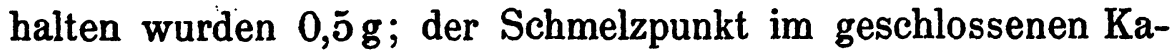


pillarrohr lag bei $186^{\circ} ; 0,1943 \mathrm{~g}$ Substanz $=24,5 \mathrm{ccm} \mathrm{N}\left(15,4^{\circ}\right.$; $749 \mathrm{~mm}$ ).

$$
\begin{array}{ll}
\mathrm{C}_{10} \mathrm{H}_{10} \mathrm{~N}_{2} \mathrm{O}_{2} . & \text { Ber.: } \mathrm{N}=14,74 \% . \\
& \text { Gef.: } \mathrm{N}=14,77 \% .
\end{array}
$$

Die beschriebenen Substanzen sind dem synthetischen Ausgangsmaterial entsprechend optiseh inaktiv. Vergleicht man mit den Eigenschaften des eben beschriebenen Anhydrids die entsprechenden des Anhydrids der Leucinursäure: große, glänzende, zu leucinartigen Schuppen vereinigte Tafeln aus Wasser; größere Wasser- und besonders leichtere Ätherlöslichkeit; Schmelzpunkt $212^{\circ}$ im geschlossenen Kapillarrohr, so ist ersichtlich, daß gegebenen Falles die Anhydrisierung der Uramidosäuren mit Erfolg zur Entscheidung herangezogen werden kann, ob Leucin oder Phenylalanin vorlag.

Galt die eingangs beschriebene und weiterhin besprochene Reaktion im besonderen dem Nachweis von Leucin, so läßt sich die Bildung von Uramidosäure wenigstens in einem gewissen Ausmaße allgemein dazu verwenden, um zu entscheiden, ob ein vorliegender Körper eine Aminosäure ist oder nicht, hauptsächlich auch dann, wenn eine nur sehr kleine Menge desselben zur Verfügung steht. Da diese Entscheidung unter Umständen von Wichtigkeit sein kann, so sei diesbezüglich eine rasch und einfach auszuführende Reaktion von genügender Empfindlichkeit mitgeteilt.

Die Grundlage dieser Reaktion bildet die schon seinerzeit mitgeteilte ${ }^{1}$ ) Beobachtung, daß Uramidosäuren und deren Salze mit salpetersaurem Quecksilberoxyd einen flockigen Niederschlag geben, der den entsprechenden Reaktionen mit Harnstoff oder Ammoniaksalzen an Empfindlichkeit nicht nachsteht, der jedoch im Gegensatz zu den letzteren, sehr ähnlichen Niederschlägen nicht nur im Überschuß der Säure, sondern auch mit derselben Leichtigkeit im Überschuß des zur Neutralisation verwendeten Alkalis löslich ist.

Die Reaktion wird in folgender Weise ausgeführt. $1-5 \mathrm{mg}$ Aminosäure werden in einer Eprouvette von den bei der eben

1) B. B., Bd. 41, S. 2953, 1908. 
Über analytische Anwendungen der Uramidosäurereaktion.

besprochenen Leucinreaktion angegebenen Dimensionen abgewogen; nach Zufügen eines entsprechenden Überschusses von Harnstoff werden 1-2 ccm kalt gesättigten Barytwassers hinzugegeben; die Eprouvette wird sodann mit einem Steigrohre in der früher angegebenen Weise versehen und deren Inhalt über einem kleinen Flämmchen etwa eine halbe Stunde im Sieden erhalten. Nach dem Abkühlen wird der Inhalt der Eprouvette in ein kleines Becherglas gespült und der Baryt mit Kohlensäure entfernt. Das Filtrat vom kohlensauren Baryt wird in einem kleinen Schälchen am Wasserbade eingedampft, der Rückstand mit einigen Tropfen Wasser aufgenommen und in 50-80 ccm einer Mischung von Alkoholäther $\mathrm{zu}$ gleichen Teilen eingetropft; nach mehrstündigem Stehen (bis der Niederschlag sich klar abgesetzt hat) wird dieser möglichst quantitativ auf ein ganz kleines dichtes Filterchen gebracht und mit der Alkoholäther-Mischung harnstofffrei gewaschen (zweckmäßig an der Saugpumpe). Der Filterrückstand wird mit wenig Wasser behandelt und das in einer kleinen Eprouvette aufgefangene klare Filtrat vorsichtig tropfenweise mit einer verdünnten Lösung von salpetersaurem Quecksilberoxyd versetzt. Die Lösung des Quecksilbersalzes soll möglichst wenig freie Säure enthalten; ein Überschuß desselben ist $\mathrm{zu}$ vermeiden; wenige Tropfen genügen, um eventuell nach Zusatz eines oder zweier Tröpfchen ganz verdünnter Lauge einen, selbst bei Verwendung von nur $1 \mathrm{mg}$ Aminosäure noch relativ dichten, weißen, flockigen Niederschlag zu erzeugen. Fährt man nun mit dem Zusatz der verdünnten Lauge vorsichtig fort, so genügen wenige Tropfen derselben, um den Niederschlag klar in Lösung zu bringen. Erst nach langem Stehen tritt infolge geringgradiger Zersetzung der Uramidosäure durch die Lauge eine geringe Trübung auf. Fällt die Probe negativ aus oder stellt man einen Blindversuch mit Harnstoff allein an, so tritt auf Zusatz von Quecksilbersalz kein Niederschlag auf; allmählicher Zusatz von Alkali ruft eine zunehmende Gelbfärbung mit entsprechender Trübung resp: Niederschlag hervor.

Es könnte der Fall eintreten, daß ein untersuchter Körper zwar nicht mit Harnstoff reagiert, wohl aber im 
letzten Filtrat erscheint und hier mit dem Quecksilbersalz einen flockigen Niederschlag gibt, eventuell wenn ein Gemisch vorlag, neben einem solchen von Uramidosäure. So weit es sich um physiologische Substanzen handelt, wird der erstere Niederschlag im allgemeinen im Überschusse der Lauge unlöslich sein. In einem solchen Falle würde man Lauge im Überschusse zusetzen, von dem unlöslichen Niederschlage abfiltrieren und das Filtrat durch vorsichtiges Ansäuren mit Salpetersäure prüfen, ob eventuell eine lösliche Quecksilberverbindung vorhanden ist oder nicht. Letzteres müßte sich dann im Überschusse der Lauge wieder lösen. Es sei diese Möglichkeit nur der Vollständigkeit halber angeführt, da dieser Fall in Wirklichkeit kaum vorkommen dürfte, um so weniger, als die in Rede stehende Reaktion, wie dies in der organischen und besonders in der physiologischen Chemie so häufig der Fall ist, nur im Zusammenhalt mit anderen Reaktionen ihren wahren Wert bekommt. Von Harnstoff und Ammonsalzen braucht hier wohl nicht weiter gesprochen zu werden, weil deren Gegenwart leicht vermieden werden kann.

Für die Säuren der Glykokollreihe ist die beschriebene Reaktion durchaus charakteristisch; das Alanin gibt zwar, wie bekannt, für sich einen Niederschlag mit salpetersaurem Quecksilberoxyd, doch ist dieser im Überschusse dar Lauge unlöslich. Tyrosin und Phenylalanin geben nach Maßgabe ihrer Löslichkeit mehr minder merkliche flockige Niederschläge mil salpetersaurem Quecksilberoxyd, die sich im Überschusse des Neutralisationsalkalis lösen; es zeigen also die freien Säuren dasselbe Verhalten wie die Uramidosäuren; wenn auch im zweiten Falle die Niederschläge dichter sein werden, so läßt sich dies keinesfalls mit Sicherheit abschätzen. Da jedoch die Uramidosäuren in Form ihrer leicht löslichen Baryumsalze zugegen sind, so muß das Filtrat neben der Quecksilberreaktion auch eine relativ starke Baryumreaktion geben, die ausbleibt, wenn man die Aminosäureprobe mit Tyrosin oder Phenylalanin allein, ohne Zusatz von Harnstoff ausführt. Bei der Asparaginsäure und Glutaminsäure, welche gleichfalls für sich schon im Überschusse des Neutralisationsalkalis lösliche Quecksilberniederschläge geben, 
läßt leider das Kriterium des Baryumniederschlages im Stiche, weil die zweite Säuregruppe Baryum festhält, so daß eine mit den beiden Säuren angestellte Aminosäureprobe, sowohl mit als auch ohne Harnstoff, ein letztes Filtrat in beiden Fällen gibt, mit welchem die Quecksilber- und die Baryumprobe positiv ausfallen. Eine Unterscheidung ist hier mit Quecksilberoxydul möglich. Ein Tropfen einer verdünnten, möglichst wenig sauern Lösung von salpetersaurem Queckssilberoxydul ruft in einer Lösung der Uramidosäuren sofort oder nach kurzer Zeit einen Niederschlag hervor, während ein solcher in einer Lösung der Aminosäuren ausbleibt.

Wenn also auch infolge der erörterten Umstände die mitgeteilte Reaktion gewiß nicht in allen Fällen für sich charakteristisch ist, so wird sie doch in vielen Fällen, schon wegen ihrer Empfindlichkeit, gute Dienste leisten können, eventuell wird sogar die Zuordnung einer Aminosäure zu einer bestimmten Gruppe möglich sein; doch sollte die Reaktion im allgemeinen nicht für sich allein, sondern als bestätigende Reaktion verwendet werden; in diesem Sinne habe ich sie hier mitgeteilt und hebe dies nochmals ausdrücklich hervor, um falschen Auslegungen vorzubeugen.

Es ist hier am Platze, noch auf eine Eigentümlichkeit der Uramidosäuren hinzuweisen, die nicht nur theoretisch interessant ist, sondern auch analytisch verwertbar sein dürfte. Wie ich schon seinerzeit mitteilte, ${ }^{1}$ ) konnte ich in einer neutralen Lösung des Natriumsalzes der Leucinsäure eine merkliche spezifische Drehung nicht konstatieren, während dasselbe Präparat nach erfolgter Anhydrisierung eine starke Linksdrehung zeigte. Ich wies auch damals darauf hin, daß diese Untersuchungen ihre Fortsetzung finden sollten, was mir leider aus äußeren Gründen bisher nicht möglich war. Inzwischen hat nun Dakin, ${ }^{2}$ ) vielleicht durch meine Mitteilung angeregt, eine Untersuchung über die spezifische Drehung der Uramidosäuren veröffentlicht. Ich möchte hier einige Daten, die schón damals gewonnen wurden, mitteilen, weil sie in mancher Beziehung

2) B. B., Bd. 41, S. 2953, 1918.

2) Amer. chem. Journ., Bd. 44, S. 48, 1910. 
interessant sind. Leucinursäure durch Kochen von optisch aktivem Hämoglobin-Leucin mit Harnstoff in reinem Wasser erhalten, wurde aus 50\%igem Alkohol umkrystallisiert. Abgewogene Mengen dieses Präparates wurden in einem bestimmten Volum rauchender Salzsäure von ca. 40\% gelöst, und das eine Mal die Drehung sofort nach erfolgter Lösung in der Kälte, das zweite Mal nach ca. einstündigem Stehen, das dritte Mal nach ca. zwölfstündigem Stehen der salzsauren Lösung in der Kälte bestimmt. Folgende Werte wurden erhalten:

1. Sofort nach Lösung; $\alpha=1,255^{\circ}, c=5,287,(\alpha)_{\mathrm{D}}^{20}=-23,19^{\circ}$

2. Nach 1 stünd. Stehen; $a=0,945^{\circ}, c=3,492,(\alpha){ }_{D}^{20}=--27,05^{\circ}$

$3 ., 12, \quad ; \alpha=2,273^{\circ}, \mathrm{c}=3,492,(\alpha)_{\mathrm{D}}^{20}=-65,08^{\circ}$

Es zeigt sich zunächst die sehr charakteristische Erscheinung, daß ein Körper, dessen spezifische Drehung so gering ist - Dakin gibt für die Leucinursäure aus l-Leucin $(\alpha)_{D}^{20}+1,9^{\circ}$ an - , daß sie bei geringerer Konzentration der Lösung (scheinbar ist auch die Reaktion derselben von Einfluß) kaum mehr sicher bestimmbar wird, durch innere Ringbildung in einen Körper von sehr viel stärkerem Rotationsvermögen übergeht. Gerade diese Erscheinung dürfte, wie mir scheint, analytisch verwertbar sein, umsomehr wenn sich herausstellen sollte, daß sie auch für andere Uramidosäuren zutrifft.

Man entnimmt ferner, wie sich das Fortschreiten der Ringbildung direkt mit dem Polarimeter verfolgen läßt, und schließlich wird wie kaum auf andere Weise deutlich, wie leicht schon in der Kälte die Wasserabspaltung resp. Hydantoinringbildung vor sich geht, allem Anschein nach erfolgt diese bei anderen Uramidosäuren z. B. solchen mit aromatischem Kern noch leichter, was seinerseits von analytischer Bedeutung ist. Der von mir für das Isobutylhydantoin in salzsaurer Lösung nach 12 stündigem Stehen gefundene Drehungswert ist nicht weit von jenem entfernt, den Dakin für denselben Körper in n-Natronlauge angibt, nämlich $(\alpha)_{D}^{20}-68,2^{\circ}$.

Vielleicht ist es nicht ohne Interesse, noch den folgenden Versuch mitzuteilen. Etwa $1 \mathrm{~g}$ der zu den mitgeteilten Drehungsbestimmungen verwendeten Leucinursäure wurde durch 
24 Stunden mit rauchender Salzsäure am Rückflußkühler gekocht; der nach Vertreiben der Salzsäure am Wasserbade hinterbliebene Rückstand wurde mit absolutem Alkohol aufgenommen; durch Einleiten von gasförmigem Ammoniak entstand ein Niederschlag, der an der Saugpumpe mit 50\%igem und 96\% igem Alkohol gewaschen wurde. Der in 20\%iger Salzsäure gelöste Körper erwies sich als optisch inaktiv. Darnach würde also bei Wiederregeneration aus der Uramidosäure auf obigem Wege das Leucin völlig razemisiert.

Zur Illustration der obigen Bemerkung, daß sich die Uramidosäuren mit aromatischem Kern besonders leicht anhydrisieren, sei als augenfälligstes Beispiel das Verhalten der Orthouramidobenzoesäure mitgeteilt. Die Anthranilsäure wurde sowohl durch Kochen mit Harnstoff und Barytwasser, als auch durch Kochen mit Harnstoff und reinem Wasser in die Uramidosäure übergeführt. Im ersten Falle entstand in dem durch Kohlensäure vom überschüssigen Baryt befreiten und etwas eingeengten Filtrat durch Salzsäure ein aus verfilzten Nadeln bestehender Niederschlag, der abgesaugt wurde. Im Filtrate davon erzeugte weiterer Salzsäurezusatz noch eine Abscheidung von Nadeln. Das Filtrat von diesen wurde neutralisiert, hierauf im Vakuum verdunstet und der Rückstand mit verdünnter Salzsäure aufgenommen, wobei sich reichlich Nadeln abschieden. Diese wurden abgesaugt und mit den ersten Fällungen zusammen aus 50\%igem Alkohol umkrystallisiert. Dabei ging ein Teil nur sehr schwierig in Lösung. Er wurde abfiltriert und bestand aus schönen langen Nadeln, deren Schmelzpunkt (im geschlossenen Kapillarrohr) bei $342^{\circ}$ lag. Das Filtrat davon gab beim Eindampfen noch eine spärliche Krystallisation des gleichen Körpers. Es wurde vorsichtig mit Salzsäure angesäuert; dabei entstand eine dichte weiße Fällung, die sich im Überschusse der Salzsäure aber alsbald wieder löste. Nach einigem Stehen schied die salzsaure Flüssigkeit reichlich lange Nadeln ab, die abgesaugt, gewaschen und unter Zusatz von etwas Tierkohle aus 50\%igem Alkohol umkrystallisiert wurden. Ihr Schmelzpunkt lag bei $340^{\circ}$. Die Stickstoff bestimmung ergab für das Hydantoin der Orthouramidobenzoesäure stimmende Werte. 
$0,1887 \mathrm{~g}$ Substanz banden $23,29 \mathrm{ccm}^{\mathrm{n}} / 10-\mathrm{H}_{2} \mathrm{SO}_{4} ; 0,1740 \mathrm{~g}$ Substanz 26,5 ccm N (20,4 $\left.{ }^{n} ; 747 \mathrm{~mm}\right)$.

$$
\begin{array}{ll}
\mathrm{C}_{8} \mathrm{H}_{6} \mathrm{~N}_{2} \mathrm{O}_{2} . & \text { Ber.: } \mathrm{N} \mathrm{17,29 \% .} \\
& \text { Gef.: N 17,28\%, 17,47\%. }
\end{array}
$$

Die eigentümliche Erscheinung des In-Lösung-Gehens des anfänglichen Niederschlages - offenbar der Uramidosäure und das nachträgliche Ausfallen des Anhydrids ist ganz analog der bei der Leucinursäure zu beobachtenden Erscheinung; nur sind hier infolge der etwas schwerer erfolgenden Wasserabspaltung die Konzentrationsverhältnisse der lösenden resp. anhydrisierenden Säure verschoben; es bedarf konzentrierter Säure zur Lösung; auch diese Lösung setzt nach längerem Stehen Krystalle von Anhydrid ab.

Mit dem bisher Mitgeteilten sind die analytischen Anwendungsmöglichkeiten der Uramidosäurereaktion keineswegs erschöpft; doch sei hier zunächst nur noch eines wichtigen Momentes gedacht.

Wohl wurde seit dem Erscheinen meiner früheren Arbeiten die Reaktion zur Isolierung von Aminosäuren empfohlen und auch mit Erfolg verwendet (Weiland, ${ }^{1}$ ) Embden und Schmitz, ${ }^{2}$ ) Kondo ${ }^{3}$ )), doch wurde dabei nur die Entstehung der Säuren im Auge behalten, die Hydantoine wurden in keinem Falle berücksichtigt. Nun ist aber gerade deren leichte Entstehung zu beachten, wenn man mit stark sauren uramidosäurehaltigen Lösungen operiert, wie es die genannten Autoren tun; anderseits erweitert sich das Anwendungsgebiet der Uramidosäurereaktion sehr bedeutend mit der Heranziehung der Hydantoine.

Denn diese Körper sind nicht nur gegen selbst höbere Mineralsäurekonzentrationen sehr resistent - wie seinerzeit schon hervorgehoben wurde und an anderer Stelle noch näher beleuchtet werden soll - , sondern sie sind zum großen Teil, im Gegensatz zu den entsprechenden Säuren, soweit in Äther löslich, daß sie schon durch Ausschütteln gewonnen werden

1) Biochem. Zeitschr., Bd. 38, S. 385, 1912.

2) Ebenda, Bd. 38, S. 393, 1912.

s) Ebenda, Bd. 38, S. 407, 1912. 
können; bei einigen derselben kommt auch die im Gegensatz zur Säure größere Schwerlöslichkeit für Wasser in Frage.

Es berührt in kritischer Beziehung sehr eigentümlich, wenn die oben genanniten Autoren, besonders Weiland und Embden, im Gegensatz zu meinen Angaben finden, daß die Uramidosäuren als solche leicht in den Äther übergehen. Sie gewinnen nämlich dieselben durch langdauernde Extraktion aus stark saurer Flüssigkeit in einem der gebräuchlichen Ätherextraktionsapparate. Einmal dürfte es jedem, der mit den genannten Apparaten gearbeitet hat, bekannt sein, daß infolge von Umständen, die hier wohl nicht erörtert $z u$ werden brauchen, Körper einer wässerigen Lösung entzogen werden können, die unter gewöhnlichen Umständen mit Äther nicht oder nur sehr schwer zu gewinnen sind; es braucht wohl kaum hervorgehoben zu werden, daß ersteres unter Umständen sehr erfreulich sein kann, daß aber hierdurch die Angaben über die praktische Löslichkeit oder Unlöslichkeit jener Körper in Äther nicht tangiert werden. Ferner muß beim Arbeiten mit Uramidosäuren in stark saurer Lösung immer mit einer eventuellen Anhydrisierung gerechnet werden, besonders bei langdauernden Extraktionen, zumal wenn sich dabei die Flüssigkeit, wie dies bei jenen Extraktionsapparaten außer unter Anwendung besonderer Maßnahmen stets der Fall ist, wenn auch gewiß nicht hochgradig, so doch merklich über Zimmertemperatur erwärmt.

Zur Illustration der wahren Löslichkeit einiger Uramidosäuren in Äther mögen die folgenden Daten dienen. $\mathrm{Zu}$ den Bestimmungen wurde gewöhnlicher stark wasserhaltiger Äther verwendet; die Säuren wurden in kleinen, mit gut eingeriebenem Stöpsel verschlossenen Fläschchen mit dem Äther zwei Stunden lang geschüttelt; es wurde sodann rasch in einen graduierten Zylinder filtriert und $20 \mathrm{ccm}$ des Filtrates in gewogenen Wägegläschen verdunstet. Bestimmt wurde die Löslichkeit für eine Leucinursäure, die aus optisch aktivem durch tryptișche Verdauung gewonnenen Casein-Leucin dargestellt worden war, sowohl durch Kochen mit Harnstoff und reinem Wasser, als auch mit Harnstoff und Barytwasser; das eine Präparạt war 
einmal, das zweite zweimal aus 50\% igem Alkohol umkrystallisiert. Ganz analog waren ferner zwei weitere Leucinursäurepräparate behandelt worden, die aus einem durch Säurespaltung aus Horn dargestellten Leucin nach den beiden Methoden gewonnen wurden. Diese vier Leucinursäurepräparate seien $\mathrm{im}$ folgenden mit $\mathrm{LC}_{1}, \mathrm{LC}_{2}, \mathrm{LH}_{1}, \mathrm{LH}_{2}$ bezeichnet; ferner wurde noch die Löslichkeit in derselben Weise für Tyrosinur- und Phenylalanursäure bestimmt. Folgende Resultate wurden erhalten (die Zahlen bedeuten $\mathrm{ccm}$ Äther):

Löslichkeit in ccm Äther: $\mathrm{LC}_{1} 1: 25000, \mathrm{LC}_{2} 1: 50000$, $\mathrm{LH}_{1} 1: 14000, \mathrm{LH}_{2} 1: 12000$, Ty $1: 3000$, Ph $1: 4000$.

Angesichts dieser Zahlen wird wohl niemand daran denken, den Äther als ein Lösungsmittel für Uramidosäuren zu bezeichnen, und es wird auch niemandem einfallen, die Ätherextraktion als ein zur Isolierung von Uramidosäuren besonders brauchbares Hilfsmittel zu empfehlen, wie die schon öfter genannten Autoren es tun. Anders natürlich wenn der Ätherextraktion eine Anhydrisierung vorausgeht. Mit Ausnahme des in Äther nur sehr wenig löslichen Hydantoins der Glykollursäure sind die Hydantoine der übrigen Glieder der Glykokollreihe in Äther relativ leicht löslich und können der wässerigen Lösung schon durch Ausschütteln um so leichter entzogen werden, je schwerer löslich sie in Wasser sind. Während das Hydantoin der Leucinursäure nach achtmaligem Ausschütteln mit Äther so gut wie quantitativ gewonnen wird, übergehen bei gleicher Zahl von Ausschüttelungen von jenem der Valinursäure etwa $80 \%$, von jenem der Alanursäure nur etwa $30 \%$. Die Hydantoine der Tyrosinur- und der Phenylalanursäure sind schwerer löslich als die vorhergehenden, doch sind sie mit Äther noch gut ausschüttelbar; infolge ihrer sehr geringen Wasserlöslichkeit kann ein großer Teil direkt gewonnen werden. Die Hydantoine der Asparaginur- und Glutaminursäure kommen für eine Isolierung durch Äther nicht in Betracht. Was die Löslichkeit der Hydantoine in anderen organischen Lösungsmiteln anlangt, so sei hervorgehoben, daß sie völlig unlöslich eigentlich nur in Petroläther sind; das Hydantoin der Tyrosinursäure ist sehr viel schwerer in Chloroform löslich als 
die meisten anderen Hydantoine, und kann daher mit Hilfe desselben von diesen getrennt werden.

Handelt es sich also darum, eine Aminosäure in dieser Form, d. h. als Hydantoin zu isolieren, und diese Form ist für viele Fälle die einzig empfehlenswerte, so wird man zunächst die Aminosäure nach einer der von mir angegebenen Methoden (welche zu wählen ist, entscheiden die näheren Umstände) in die Uramidosäure überführen. Im Prinzip handelt es sich nun weiter darum, die Uramidosäure ohne vorhergehende Isolierung in das Hydantoin umzuwandeln. Nach meinen Methoden liegt sie entweder als Barytsalz oder als Ammonsalz vor und zwar im allgemeinen in einer Lösung, die eine größere oder geringere Menge Harnstoff, und eventuell noch andere Bestandteile, wie Salze in größerer Menge usw., enthält. Man wird nun je nach der weiteren Verwendung der zu behandelnden Lösung dieselbe mit Salzsäure oder mit Schwefelsäure zum Sieden erhitzen, im letzteren Falle nachdem man von eventuell ausgefallenem Baryumsulfat abfiltriert hat. Es wird im allgemeinen mehr Säure anzuwenden sein als für reine Uramidosäurelösungen, einmal weil etwa vorhandene Salze die Dissoziation der verwendeten Säure zurückdrängen können, und dann, weil ein Teil der Säure zur Zersetzung des überschüssigen Harnstoffs verbraucht wird; es wird daher notwendig sein, wenn man nicht von vornherein einen allzugroßen Säureüberschuß zusetzen will, von Zeit zu Zeit die Reaktion der kochenden Flüssigkeit zu prüfen, bis keine Abnahme der sauren Reaktion mehr erfolgt. Über die Dauer des Kochens lassen sich unter diesen Umständen bestimmte Zeitangaben nicht machen, doch soll dasselbe, zumal bei höherer Säurekonzentration nicht zu lange ausgedehnt werden, weil man sonst trotz der Resistenz der Hydantoine gegen Säuren Gefahr läuft, einen, wenn auch relativ geringen Teil derselben zu zersetzen. Nach dem Erkalten filtriert man von dem eventuell ausgefallenen Teil des Hydantoins ab und schüttelt mit Äther aus, oder extrahiert in einem der bekannten Extraktionsapparate. Besonders im letzteren Falle tut man gut, den Äther vor dem Abdampfen zu entwässern. Stehen größere Mengen Material zur Verfügung, 
so kann man das gewonnene Hydantoin zur weiteren Bestätigung durch Kochen mit Barytwasser in die entsprechende Uramidosäure zurückverwandeln.

$\mathrm{Da} B$ in dieser Form die Methode einen genügend allgemeinen Grad von Anwendbarkeit besitzt und einen wirklichen Fortschritt gegenüber den bisher gebräuchlichen Methoden zur Isolierung von Aminosäuren besonders für physiologische Zwecke darstellt, davon kann sich jeder überzeugen, der in die Lage kommt, Aminosäuren unter verschiedenen Umständen $\mathrm{zu}$ isolieren und identifizieren zu müssen, besonders wenn es sich um kleine Mengen handelt. Die Methode kann bis zu einem gewissen Grade auch zur Trennung von Aminosäuren verwendet werden; inwieweit sie für sich oder in Kombination mit der Abscheidung der Uramidosäuren als solche zur Auflösung eines Aminosäuregemisches in seine Komponenten dienen kann, darüber sind die Untersuchungen noch nicht abgeschlossen; sicher kann die Methode bei der Zerlegung mancher Esterfraktionen gute Dienste leisten. Auch in quantitativer Hinsicht läßt sich sowohl die Uramidosäurereaktion im allgemeinen, als auch in Form der Hydantoinreaktion verwenden usw. Einiges von dem bisher gewonnenen Material soll in weiteren Mitteilungen vorgelegt werden, doch befindet sich der Gegenstand noch nach jeder Richtung hin in weiterer Bearbeitung.

Im folgenden seien noch einige Daten über zwei bisher noch nicht beschriebene Hydantoine mitgeteilt.

Das Anhydrid oder Hydantoin der Valinur- resp. $\alpha$-Uramido-isovaleriansäure wurde durch Kochen von inaktiver Säure mit n/4-Schwefelsäure. am Rückflußkühler erhalten. Nach dem Erkalten der Flüssigkeit setzten sich keine Krystalle ab. Die mit Baryumacetat genau ausgefällte Lösung wurde nach dem Filtrieren am Wasserbade eingedampft; der Rückstand ging relativ leicht in Äther über, nach dem Verdunsten desselben hinterblieb das Anhydrid in Form großer weißer, glänzender, zu Gruppen vereinigter rhombischer Tafeln. Diese lösen sich ziemlich leicht in Wasser, Alkohol und Äther. Ihr Schmelz. punkt im geschlossenen Kapillarrohr liegt bei $132^{\circ}$. 0,1359 g Substanz banden $18,88 \mathrm{ccm} \mathrm{n/10-} \mathrm{H}_{2} \mathrm{SO}_{4}$. 
Über analytische Anwendungen der Uramidosäurereaktion.

$$
\mathrm{C}_{6} \mathrm{H}_{10} \mathrm{~N}_{2} \mathrm{O}_{2} \text {. Ber.: } \mathrm{N}=19,72 \%
$$

Das Anhydrid oder Hydantoin der $\alpha$-Uramidoglutarsäure resp. Glutaminursäure wurde gleichfalls durch Kochen der Säure mit n/4-Schwéfelsäure am Rückflußkühler dargestellt. Nach genauer Ausfällung mit Baryt wurde am Wasserbade eingedampft; über konzentrierter Schwefelsäure wurde der Rückstand krystallinisch. Er löste sich leicht in, wässerigem Alkohol; auf Zusatz von Aceton entstand eine flockige Fällung, die krystallinisch wurde; beim langsamen Abdunsten der Flüssigkeit bildeten sich große flache sechsseitige tafelförmige Krystalle. Diese wurden in absolutem Alkohol gelöst; nach einigem Stehen schieden sich sechsseitige Tafeln ab, die langsam zu größeren Krystallen und Drusen heranwuchsen; beim rascheren Verdunsten des Alkohols bildeten sich große prysmatische Krystalle und prysmatische Nadeln. Das Hydantoin ist leicht löslich in Wasser, ziemlich löslich in Alkohol, kaum löslich in Äther. Sein Schmelzpunkt lag im geschlossenen Kapillarrohr bei $168^{\circ}$. 0,2109 g Substanz 30,4 ccm N (16 $\left.{ }^{\circ} ; 733,1 \mathrm{~mm}\right)$.

$$
\begin{array}{ll}
\mathrm{C}_{6} \mathrm{H}_{8} \mathrm{~N}_{2} \mathrm{O}_{4} . & \text { Ber.: } \mathrm{N}=16,28 \% \\
& \text { Gef.: } \mathrm{N}=16,49 \% .
\end{array}
$$

Man wird bemerkt haben, daß ich in der vorliegenden Abhandlung bestrebt war, bezüglich der Uramidosäuren eine möglichst einheitliche Nomenklatur anzuwenden. Es zeigte sich hierzu die alte Namenbildung durch Anhängung der Silbe Uran den Namen der betreffenden Aminosäure am geeignetsten. Da es sich um Körper handelt, deren Namen wahrscheinlich in der Folgezeit häufiger gebraucht werden dürften, so erlaube ich mir, aus Zweckmäßigkeitsrücksichten vorzuschlagen, auch die Anhydride oder Hydantoine in diese einheitliche Nomenklatur mit einzubeziehen in der Weise, daß dem mit der Silbe Ur- verbundenen Namen der Aminosäure noch die von dem Worte Anhydrid oder Hydantoin genommenen Endsilben angehängt werden. Es würden also darnach beispielswëise die zugehörigen Bezeichnungen zu bilden sein:

Leucin, Leucinursäure, Leucinurid oder Leucinuroin. 
144 F. Lippich, Analytische Anwendungen der Uramidosäurereaktion.

Phenylalanin, Phenylalanursäure, Phenylalanurid oder Phenylalanuroin.

Diese Zusammenstellungen zeigen wohl die Übersichtlichkeit und Zweckmäßigkeit der vorgeschlagenen Nomenklatur wenigstens vom praktischen Standpunkte, der ja häufig genug neben dem unvermeidlich schwerfälligen rein chemischen zu Worte kommt. 